\title{
Foetal airway motor tone in prenatal lung development of the pig
}

\author{
M.P. Sparrow, S.P. Warwick, H.W. Mitchell
}

\begin{abstract}
Foetal airway motor tone in prenatal lung development of the pig. M.P. Sparrow, S.P. Warwick, H.W. Mitchell. OERS Journals Ltd 1994.

ABSTRACT: The terminal airways from embryonic lung in situ or as explants exhibit rhythmic spontaneous contractions. Our objective was to see whether narrowing responses of the airways occurred throughout the bronchial tree in the first trimester foetus and, if so, to characterize them.

The bronchial tree was freed of vasculature and parenchyma from the lungs of 20-35 g pig foetuses (44-48 days gestation). The airway lumen was visualized directly with transmitted light, and narrowing was recorded in real time by video-imaging microscopy.

From the main stem bronchi to the terminal regions of late generation branches (20-35 $\mu \mathrm{m}$ i.d.) strong bronchoconstrictor responses to micromolar concentrations of acetylcholine (ACh), histamine, substance $\mathbf{P}$ and $\mathrm{K}^{+}$depolarizing solution were seen, whilst inhibition of narrowing with $\beta$-adrenoceptor agonists was evidence of $\beta$-receptors on the smooth muscle. Moreover, strong narrowing responses to electrical field stimulation, which were blocked by atropine, indicated that functional cholinergic nerves were present. A remarkable display of spontaneous narrowing in the airways of many of the bronchial tree preparations caused the movement of lung liquid to and fro.

We speculate that the bronchomotor tone and associated spontaneous activity, which move the lung fluid along the airways, serve to maintain an even positive pressure in localized areas of the bronchial tree which is essential to provide the stimulus for continued growth of the lung.

Eur Respir J., 1994, 7, 1416-1424.
\end{abstract}

Dept of Physiology, University of Western Australia, Nedlands, Australia.

Correspondence: M.P. Sparrow Dept of Physiology

University of Western Australia Nedlands 6009

Australia

Keywords: Airway narrowing bronchial tree

bronchomotor tone

lung liquid

pig foetal airways

spontaneous narrowing

Received: December 141993

Accepted after revision March 301994

This research was supported by the National SIDS Foundation of Australia and the Raine Medical Foundation of Western Australia.
The normal growth of the foetal lung depends on pressures acting across the lungs, that are maintained by the volume of fluid in the airways and by the resistance of the upper airway to fluid efflux [1]. The periodic opening of the latter drains excess lung liquid, thereby regulating lung volume [1]. However, whether the lower airways contribute to lung tissue pressure by exerting bronchomotor tone is unknown.

The functional maturity of the airway smooth muscle in foetal pigs has been studied at various ages of foetal development, using the trachealis [2]. The maximum isometric force generated in response to different modes of stimulation, including electrical field stimulation (EFS), when normalized as stress (force/cross sectional area of smooth muscle cells), was similar in 9-16 g foetal pigs ( $\sim 36-41$ days gestation) to that in late term foetuses (full term 115 days, weight $1,100 \mathrm{~g}$ ). The level of maturity of the bronchial smooth muscle in the growing airways has not been studied. However, rhythmic spontaneous contractions of airways in explants of lung from chick embryos were reported early this century by LEwIS [3] and later by SCHOPPER [4,5] using cultures of lung from guinea-pig. Very recently, MCCRAY [6] noted spontaneous activity of the epithelial tubes in parenchymal lung tissue from first trimester human foetuses. After culturing explants for $48 \mathrm{~h}$, growing epithelial tubes were observed to narrow both spontaneously and to a variety of smooth muscle stimulants.

The bronchial tree develops from the primitive epithelial tube, an outgrowth from the foregut, which undergoes extensive branching morphogenesis within the mesenchyme [7], with differentiation of the smooth muscle cells arising through the tissue-specific mesenchyme/epithelial cell interaction [8,9]. Presumptive smooth muscle cells, i.e. cells staining with smooth muscle $\alpha$-actin, are first located in the clefts of the bifurcating epithelial tubes, followed by smooth muscle myosin and desmin staining as the airways enlarge in 17-19 day foetal rats $[10,11]$.

It seemed likely from the above observations that the bronchial smooth muscle and its neurotransmission may well be functional in the bronchial tree, but the extent to which this occurred needed to be determined. Since it was not very feasible to quantitate smooth muscle contractility directly in distal airways as small as $20 \mu \mathrm{m}$ lumen diameter, we determined the capacity of the airways to narrow. Narrowing of the airways throughout the bronchial tree was visualized directly by 
video-imaging microscopy, so that spontaneous narrowing and the movement of lung liquid could be recorded in real time. In addition, responses were quantified both to direct smooth muscle stimulation using agents such as acetylcholine (ACh), histamine and substance $\mathrm{P}$ and indirectly via the nerves in the airway wall.

\section{Methods}

Foetal pigs were obtained from a local abattoir. They were hybrids from Large White/Landrace sows against first cross Large White/Landrace boars. After removal from the uterus approximately 20 min after death of the sow, they were packed on ice and transported back to

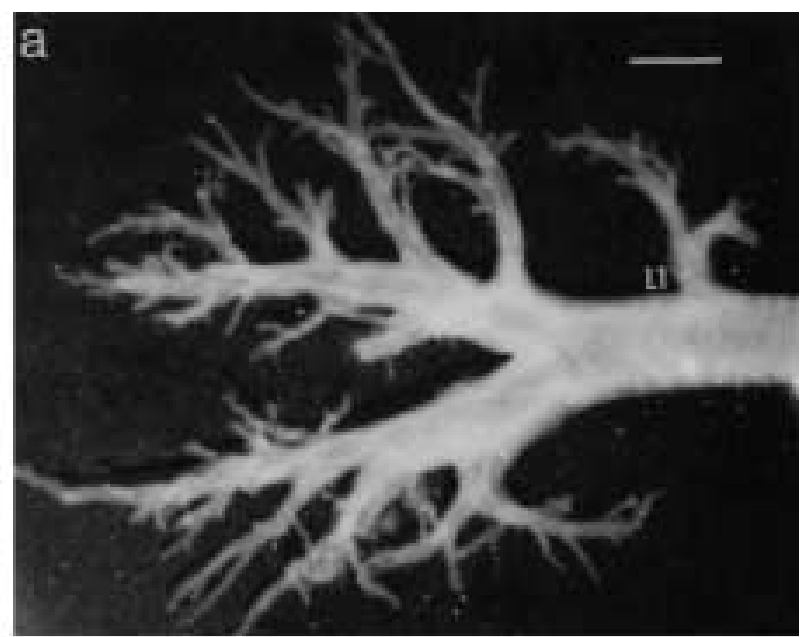

b)

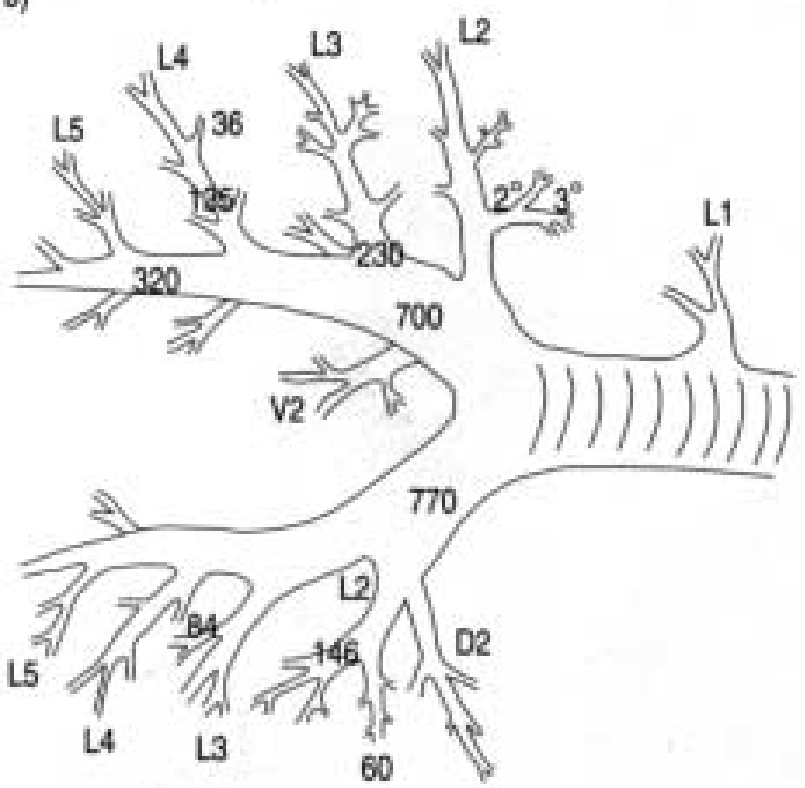

Fig. 1. - a) a dorsal view of the bronchial tree from the lung of a $20 \mathrm{~g}$ foetal pig after removal of the parenchyma and vasculature. The first lateral (L1) on the right-hand side buds off the trachea. There is no equivalent lateral on the left hand side. (Scale bar 1,000 $\mu \mathrm{m})$. b) A schematic drawing of the same bronchial tree showing the disposition of the laterals (L), dorsals (D) and ventrals (V) and the second $\left(2^{\circ}\right)$ and third $\left(3^{\circ}\right)$ order branches used in this study, with dimensions $(\mu \mathrm{m})$ to indicate lumen diameter. the laboratory. The body weight and crown-rump length were measured before removal of the trachea and lungs. Foetuses weighed between 20-35 g with a crown-rump length of $70-85 \mathrm{~mm}$, which we estimate is approximately 44-48 days gestation, according to published data [12, 13]. This length of gestation is toward the end of the first foetal stage [13] in the pig, and comparable with the mid-metamorphosing stage in humans (crown-heel length $\sim 15 \mathrm{~mm}$ ). Using a dissecting microscope at $8-16 \times$ magnification, the parenchyma and vasculature were carefully teased away with jeweller's forceps (50 $\mu \mathrm{m}$ tips) from the bronchial tree, which was kept immersed in cold Krebs solution (fig. 1). It was then placed in a glass bath coated on the bottom with Sylgard 184 silicone elastomer (Dow Corning, Michigan, USA) to form a chamber of $5 \mathrm{ml}$ volume, through which a steady flow of Krebs solution $\left(5-10 \mathrm{ml} \cdot \mathrm{min}^{-1}\right)$ at $32^{\circ} \mathrm{C}$ was perfused, and kept in place with a Minutien pin $100 \mu \mathrm{m}$ diameter (Fine Science Tools Inc., Vancouver, BC Canada) through the proximal trachea. A video-image of the bronchial tree was obtained with a colour CCTV camera (Panasonic CL700) attached to a dissecting microscope, using a suitable intensity of transmitted light. The image was displayed on a colour video-monitor, and changes in the airway lumen diameter due to contractile activity were recorded in real time on a Super-VHS video-recorder (Panasonic NV-FS90 HQ) at a total magnification of $40-80 \times$ for large airways and at $160 \times$ for the smallest airways. Drugs were added to the Krebs solution perfusing over the preparation and the response monitored until a steady-state of narrowing (or relaxation) was achieved, at which point a higher concentration was perfused if cumulative concentration-response curves were required. EFS was carried out with insulated platinum or silver wire electrodes, $250 \mu \mathrm{m}$ diameter, with the tips exposed and mounted on a micromanipulator. At the end of an experiment, the preparation was fixed by the addition of $4 \%$ formaldehyde to the Krebs solution perfusing the bath. For cryostat sections, it was removed, placed in Cryo-M-Bed and cooled to $-196^{\circ} \mathrm{C}$ in isopentane precooled in liquid nitrogen.

\section{Video-imaging procedure and analysis}

To observe narrowing of the whole bronchial tree, the lumen was most readily visualized by gently perfusing Krebs solution, tinted blue-black with Amidoschwarz 10 B (Merck) dye, through the trachea to highlight the bronchial lumen. The distal ends of the main stem bronchi and some of the early laterals (L2-4) were severed to enable, as much as possible, the free passage of Krebs solution through the bronchial tree. When viewed under dark-field illumination, this produced a striking contrast between the bronchial wall and the lumen (fig. 2a). The use of transmitted light with some added sidelighting also produced a very good contrast with the tinted Krebs solution. However, most of the experiments were performed at higher magnification $(40-160 \times)$, where it was unnecessary to use the tinted Krebs solution in the lumen, i.e. the intact bronchial tree was used without disturbing 

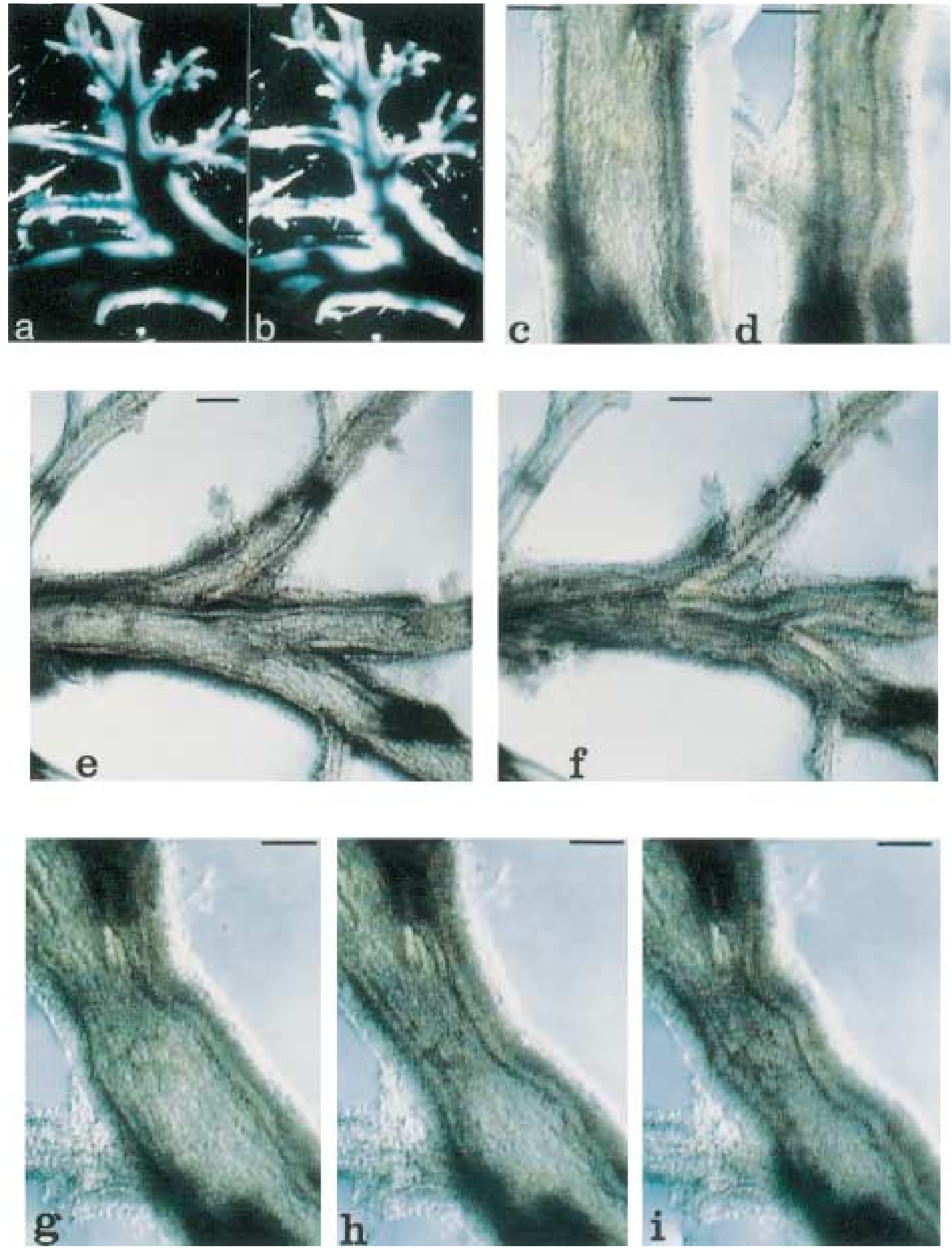

Fig. 2. - Video-images of narrowing responses of the bronchial tree from a $20 \mathrm{~g}$ foetal pig lung viewed (a and b) under dark-field illumination, with the lumen highlighted by perfusing it with blue-tinted Krebs solution; or (c-i) using transmitted light at higher magnification, 80-160×, without intervention, so that the lung liquid could be seen moving in the lumen. a) Relaxed; and b) maximal narrowing after stimulation with acetylcholine (ACh) $100 \mu \mathrm{M}$ in the bathing solution. (Scale bar=500 $\mu \mathrm{m}$ ). c and d) A $360 \mu \mathrm{m}$ lumen diameter relaxed airway before and after narrowing to ACh $100 \mu \mathrm{M}$, where the lumen narrows and wall thickens. e and f) Lateral L2 right hand side (RHS) with branches from $35 \mathrm{~g}$ foetal pig: e) relaxed; and f) narrowed after electrical field stimulation $(0.1 \mathrm{~ms}, 15 \mathrm{~Hz}, 20 \mathrm{~V}) . \mathrm{g}-\mathrm{i})$ Examples of spontaneous narrowing of a lateral, $350 \mu \mathrm{m}$ i.d., which are taken from a sequence of spontaneous activity. (Scale bar $(c-i)=200 \mu \mathrm{m})$. 
the lumen contents. All the quantitative data reported here were obtained in this way. A clearly defined airway wall and lumen could readily be obtained using transmitted light (fig. 2c-i) in most airways. However, the proximal stem bronchus was not suitable for measuring outside diameter because the outer edge was indistinct, presumably due to the condensed, thickened, extracellular matrix adhering to it. Also, the close proximity of the exit points of the lateral, ventral and dorsal branches leading off the proximal stem bronchus greatly restricted the number of sites where quantitation of lumen diameter by video-imaging was possible.

After video-recording an experiment, suitable sections of airways were displayed and the lumen and external diameter were quantified using Chromatic Colour Image Analysis Software (Leading Edge, South Australia) by using a cursor to draw a line transversally across the airway. At least three measurements were made at each site for large airways, six for small and nine for airways $<100 \mu \mathrm{m}$. Usually, a site was chosen midway between branches. To find the same location when comparing the dimensions in a contracted and relaxed airway, a prominent site, e.g. a branching point, was identified as a reference feature. At maximum magnification $(160 \times)$ on the computer screen, the coefficient of variation for nine measurements of the lumen diameter of an airway maximally narrowed to $\sim 100 \mu \mathrm{m}$ i.d. was $5 \%$, and similarly for an airway narrowed to $25 \mu \mathrm{m}$ i.d. was $6.8 \%$. At maximum magnification, one pixel was equal to $4 \mu \mathrm{m}$.

To check the reliability of the visually-observed measurement of airway wall thickness and lumen diameter during narrowing, the wall area was calculated before and after maximal narrowing using the lumen and external diameter measurements. There was no significant change in wall area during narrowing (paired t-test), and a regression line fit for linearity gave a coefficient of determination $\left(\mathrm{R}^{2}\right)$ of $0.97,(\mathrm{p}<0.001)$ between contracted and relaxed wall area, with a slope of 0.99 . Furthermore, these parameters compared closely with those obtained from haemotoxylin and eosin $(\mathrm{H} \& \mathrm{E})$ stained sections obtained by cryosection. The wall area of the relaxed airway calculated from imaging measurements of the lumen and external diameter was highly correlated with that obtained by morphometry of the H\&E stained airway $\left(\mathrm{p}<0.001 ; \mathrm{R}^{2}=0.99\right)$, and the slope of the regression line was 0.96 . For this morphometry the formaldehyde was washed out from the fixed airways, frozen in Cryo$\mathrm{M}-\mathrm{Bed}$, and $10 \mu \mathrm{m}$ sections cut and H\&E stained. Area measurements of the lumen and the total area to the outside edge of the smooth muscle were obtained using colour discrimination and the wall area calculated.

\section{Nomenclature of the airways}

Airways were designated according to their sequence of development, based on the nomenclature of FLINT [7]. Figure 1a shows a dorsal view of the bronchial tree of a $20 \mathrm{~g}$ foetal pig. The first lateral (L1) on the right-hand side buds off the trachea. There is no equivalent L1 on the left-hand side. Figure $1 \mathrm{~b}$ shows the disposition of the laterals (L), dorsals (D) and ventrals (V) used in the study, with dimensions $(\mu \mathrm{m})$ to indicate relaxed lumen diameter (i.d.). In $35 \mathrm{~g}$ foetal pigs, the airways were correspondingly larger, e.g. the base of the stem bronchus was $\sim 1,000 \mu \mathrm{m}$ i.d. In general, quantitative recordings were chiefly from the laterals L2, 3 and 4 and V2 righthand side (RHS) and L2, 3 and D2 (left hand side (LHS)). The proximal set of branches arising from a lateral or dorsal were designated 2 nd order branches $\left(2^{\circ}\right)$; the next more distal set $\left(3^{\circ}\right)$; and so on. The airways used were grouped according to their size and designated "large", "medium", "small", "smaller" and "smallest" and their location as shown in table 1. For example, a $300 \mu \mathrm{m}$ i.d. airway (category "small") was taken only from L2, 3 or V2 and D2, although it could feasibly have been from the distal end of the stem bronchus. However, only the former were included in the study in an attempt to

Table 1. - The characteristics of the airways of the bronchial tree from 20-35 $\mathrm{g}$ foetal pigs shown in the categories used to study function

\begin{tabular}{|c|c|c|c|c|c|c|}
\hline Category & Location & $\begin{array}{l}\text { Size range (i.d.) } \\
\mu \mathrm{m}\end{array}$ & $\begin{array}{l}\text { Narrowing } \\
\% \text { of i.d. }\end{array}$ & $\begin{array}{c}\mathrm{EC}_{50} \\
\mu \mathrm{M}\end{array}$ & $\begin{array}{c}\text { Wall } \\
\text { thickness } \\
\mu \mathrm{m}\end{array}$ & $\begin{array}{c}\text { Increase } \\
\text { in thickness } \\
\%\end{array}$ \\
\hline Large & $\begin{array}{l}\text { Stem bronchi } \\
\text { (proximal) }\end{array}$ & $800-950$ & $\begin{array}{c}38 \pm 1.4(5) \\
*\end{array}$ & $\begin{array}{c}8.2 \\
(7.2-9.4)\end{array}$ & - & - \\
\hline Medium & $\begin{array}{l}\text { Stem bronchi } \\
\text { (mid/distal) }\end{array}$ & $531-645$ & $\begin{array}{c}26 \pm 1.6(8) \\
* *\end{array}$ & $\begin{array}{c}5.1 \\
(3.2-8.1)\end{array}$ & $29 \pm 1.6$ & $127 \pm 10.3$ \\
\hline Small & $\begin{array}{l}\text { L2, 3, V2 RHS } \\
\text { L2, 3, D2 LHS }\end{array}$ & $184-350$ & $18 \pm 1.3(8)$ & $\begin{array}{c}0.27 \\
(0.23-0.32)\end{array}$ & $24 \pm 1.0$ & $86 \pm 14.1$ \\
\hline Smaller & L4 RHS \& LHS & $60-130$ & $23 \pm 1.5(9)$ & - & $18 \pm 0.9$ & $93 \pm 4.4$ \\
\hline Smallest & $2^{\circ} \mathrm{L} 4$ or $3^{\circ} \mathrm{L} 3$ & $22-45$ & $27 \pm 2.1(9)$ & - & - & - \\
\hline
\end{tabular}

The maximum narrowing, increase in wall thickness and $\mathrm{EC}_{50}$ to acetylcholine are shown. Narrowing is expressed as percentage of relaxed lumen diameter (i.d.) Data are presented as mean \pm SEM. Number of bronchial tree preperations shown in parenthesis. $\mathrm{EC}_{50}$ is shown with a range in parenthesis corresponding to \pm 1 SEM calculated from the $\mathrm{pD}_{2}$. Significance of difference of the means for narrowing: *: large $v s$ medium $(\mathrm{p}<0.001)$; **: medium $v s$ small $(\mathrm{p}<0.002)$. L: lateral; $\mathrm{D}:$ dorsal; $\mathrm{V}:$ ventral; $2^{\circ}$ : secondary; $3^{\circ}$ : tertiary; RHS: right-hand side; LHS: left-hand side (see fig. 1). $\mathrm{EC}_{50}$ : concentration producing half the maximal effect. 
preserve, where possible, homogeneity with respect to the developmental maturity of airways.

\section{Immunocytochemistry and histology}

Immunocytochemical procedures were as described by Воотн et al. [2]. At the end of an experiment, the bronchial tree was placed in Cryo-M-Bed and frozen in isopentane precooled in liquid nitrogen. Transverse sections of airways, $10 \mu \mathrm{m}$ thick, were cut on a cryostat and stained concurrently for both smooth muscle myosin, using a polyclonal antibody to smooth muscle myosin [14], followed by a rhodamine red conjugated secondary antibody, and for nerve fibres using a monoclonal antibody to the $68 \mathrm{kDa}$ component of neurofilament (Amersham, UK) followed by a fluorescein isothiocyanate (FITC) conjugated secondary antibody. For H\&E staining, the bronchial tree was fixed in formal saline (4\% formaldehyde) in situ at the end of an experiment. For routine examination, paraffin embedding was used with $5 \mu \mathrm{m}$ sections.

\section{Statistics}

Data are shown as the mean \pm SEM. The significance of the difference between the means of two groups of data was assessed using the Student's t-test for unpaired and paired groups, with $\mathrm{p}<0.05$ considered significant. For more than two sets of data, an analysis of variance was used followed by a Newman-Keuls Multiple Comparisons Test. The number ( $\mathrm{n}$ ) of bronchial tree preparations used varied from five to eight. The concentration producing half the maximal effect $\left(\mathrm{EC}_{50}\right) \pm$ SEM is shown as the geometric mean with the range calculated from \pm 1 SEM of the mean of the $\mathrm{pD}_{2}$ (which is $\left.\log \mathrm{EC}_{50}\right)$.

\section{Results}

For the purposes of viewing overall narrowing throughout the whole bronchial tree, dark-field illumination was used, with the lumen highlighted with Krebs solution tinted blue-black (fig. 2a). When maximal ACh $100 \mu \mathrm{M}$, was delivered in the Krebs solution flowing over the bronchial tree, the laterals began to narrow within $10 \mathrm{~s}$ of contact, and maximal narrowing of the lumen to give a threadlike appearance was achieved in $30 \mathrm{~s}$ (fig. 2b). The proximal region of the stem bronchi narrowed considerably less and took up to $60 \mathrm{~s}$ for maximum narrowing. As it began to narrow, the distal end and its side branches elongated, presumably due to the pressure building up in the lumen of the stem bronchus, thereby forcing lung fluid peripherally into the more distal airways, which were also constricting or fully constricted. Relaxation followed after withdrawal of the ACh from the perfusate in $<30 \mathrm{~s}$ in the second order branches, and up to $100 \mathrm{~s}$ in the stem bronchi. This delay may have been due to the thicker wall of the stem bronchus and the greater amount of condensed extracellular matrix adhering to it. ACh was also perfused through the lumen with similar narrowing responses observed.

For quantitative purposes, e.g. obtaining concentrationnarrowing response curves, individual bronchi of the intact bronchial tree could be clearly focused using transmitted light at higher power, thereby eliminating the need for the tinted Krebs solution in the lumen. Thus, the lung liquid present in the lumen of the foetal airway could be observed in situ. The wall of short sections of bronchi, which were relatively free of branches and which lay in the horizontal plane, could be clearly defined (fig. $2 \mathrm{c}-\mathrm{i}$ ), enabling the internal and external dimensions of the airway to be measured (see Methods). The relaxed airway wall became thinner as the airways decreased in size (table 1). During narrowing, the wall thickened progressively and at maximum narrowing elicited with ACh was approximately doubled (fig. 2c-f and table 1). This appeared to be due to the increased folding of the mucosa encroaching into the lumen which was seen on examination of histological sections (see below).

\section{Responsiveness of airways}

The responsiveness of airways to ACh is listed in table 1 , according to size and location (see Methods) (fig. 1) and gives their reactivity and, where feasible, their sensitivity. Figure 3 shows that small bronchioles narrowed significantly more than the large (proximal stem bronchi),

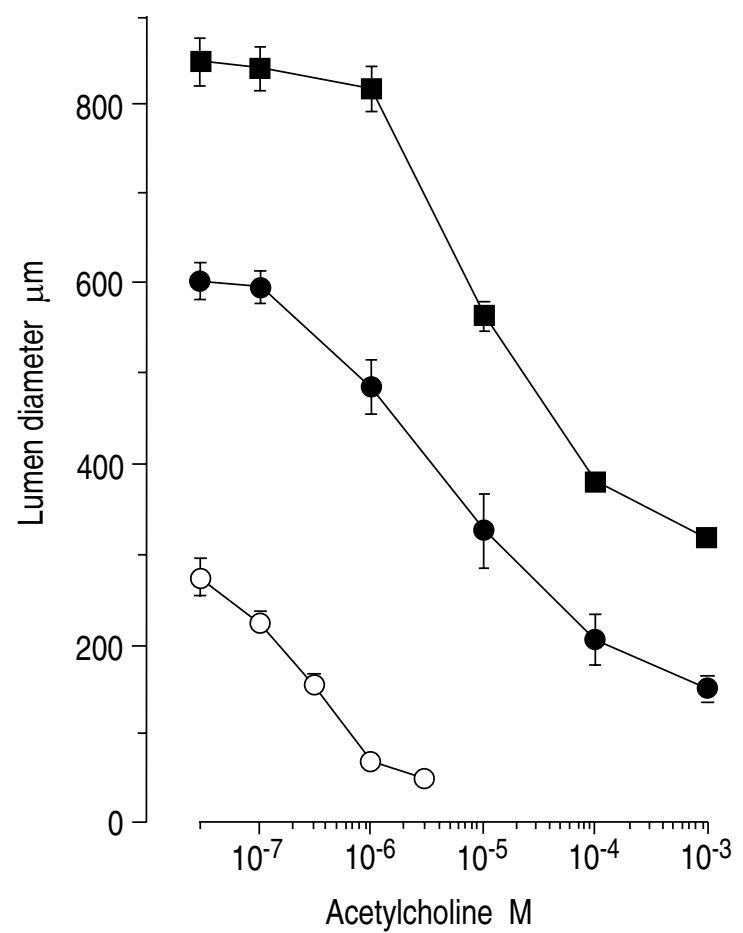

Fig. 3. - Concentration-narrowing response characteristics to acetylcholine (ACh) of large (- - ), medium (-) and small (-O) diameter airways from the bronchial tree of foetal pigs. The small airways are most sensitive and narrow to the greatest extent. Data points are means with the vertical bars showing ISEM where they are larger than the symbol. Airway sizes are shown in table 1 . 
a)

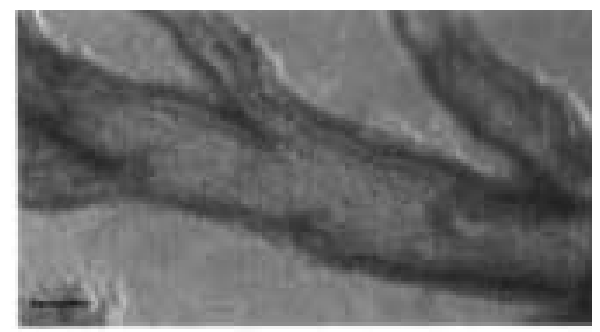

b)

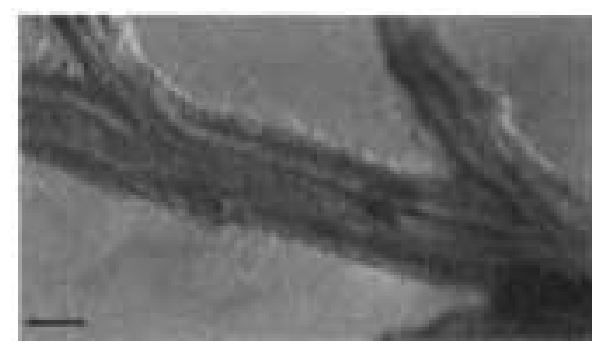

c)

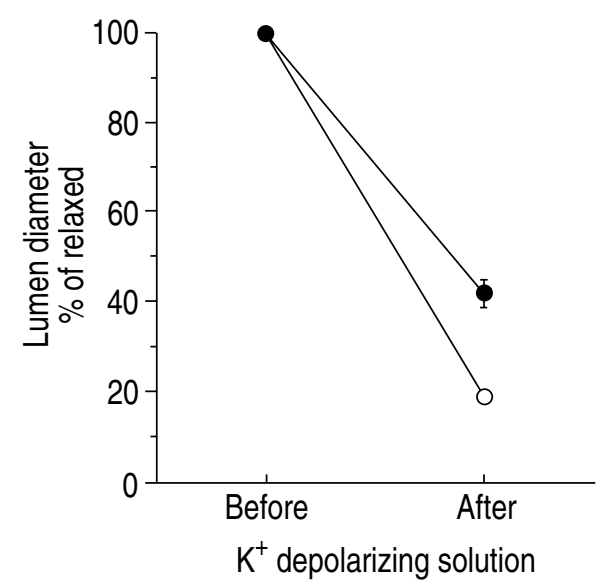

Fig. 4. - Narrowing responses to $\mathrm{K}^{+}$depolarizing solution in a lateral (300 $\mu \mathrm{m}$ i.d.) and its side branches (70 and $125 \mu \mathrm{m})$. a) Relaxed; b) narrowed; c) the extent of narrowing compared in large $(-\mathbf{-})$ and small $\left(-\mathrm{O}_{-}\right)$airways. The lumen diameter has been expressed as percentage of relaxed lumen diameter. The small airways narrow significantly more $(\mathrm{p}<0.001)$. (Scale bar $=200 \mu \mathrm{m})$.

to 18 vs $36 \%$, respectively, of relaxed i.d. $(\mathrm{p}<0.001)$, and were more sensitive. The threshold for small was $<0.1$ $\mu \mathrm{M}$ and for large $>1 \mu \mathrm{M}$, and the former was 27 times more sensitive at their $\mathrm{EC}_{50}(\mathrm{p}<0.001)$. The medium group (mid to distal region of the stem bronchi) was intermediate in its reactivity and sensitivity. With smaller airways, $<130 \mu \mathrm{m}$, narrowing was measured only to a single maximum concentration of ACh $(10 \mu \mathrm{M})$, above which no further increase in reactivity was seen (table 1). This was because it was not possible to measure with sufficient accuracy the changes in lumen diameter to incremental increases of ACh. Atropine, $0.1-1 \mu \mathrm{M}$, completely blocked these bronchoconstrictor responses.

With depolarizing solution (high $\mathrm{K}^{+}$) small airways narrowed more than large. The lumen was reduced to $18 \pm 1.6 \%(n=6)$ of relaxed i.d., compared with $41 \pm 3.0 \%$ $(n=7)$ in large $(p<0.001)$ (fig. 4). Histamine and substance $\mathrm{P}$ were also effective bronchoconstrictors of the bronchial tree. In small airways, the $\mathrm{EC}_{50}$ for histamine was $0.6 \mu \mathrm{M}(0.59-0.71 \mu \mathrm{m})$, and the maximal narrowing at $10 \mu \mathrm{M}$ was $33 \pm 3 \%(\mathrm{n}=7)$. Substance P narrowed small airways by $50 \%$ at $0.1 \mu \mathrm{M}$, and at $10 \mu \mathrm{M}$ narrowed the airways maximally to $19 \pm 3 \%(n=4)$ of initial lumen diameter. The $\beta$-adrenoceptor agonist, isoprenaline, substantially relaxed the bronchial tree. In small airways previously constricted with $1 \mu \mathrm{M}$ ACh to $40 \%(n=3)$ of relaxed i.d., $100 \mu \mathrm{M}$ isoprenaline produced near complete relaxation in $<5 \mathrm{~min}$, but in preparations maximally contracted with $10 \mu \mathrm{M}$ ACh $(n=3)$ it was less effective, producing only partial relaxation (to $62 \pm 4.4 \%$ of relaxed i.d.) after 5 min.

Sometimes, the foetal airways displayed spontaneous narrowing and relaxation, which was seen throughout most of the bronchial tree as irregular patches of activity (fig. $2 \mathrm{~g}-\mathrm{i}$ ). These varied from localized movements of the wall in some foetal pigs to strong contractions in others. On some occasions, these commenced as peristaltic-like waves moving in a distal to proximal direction over small distances $\sim 2 \mathrm{~mm}$, with the waves of contraction increasing in complexity. Most often, the complex contraction pattern showed no definite direction. At times, strong continuous activity over a $\sim 3 \mathrm{~mm}$ length of bronchus was recorded. These spontaneous contractions were largely blocked by verapamil $10 \mu \mathrm{M}$, and completely blocked at $100 \mu \mathrm{M}$.

A striking observation was the movement of lung liquid through the airways during narrowing. This movement was detected by the cellular debris that it carried, which was sometimes in loose clusters but more often dispersed finely throughout the fluid. The small size of the particles $(<4 \mu \mathrm{m})$ and their poor contrast against the background of the airway wall prevented them from being

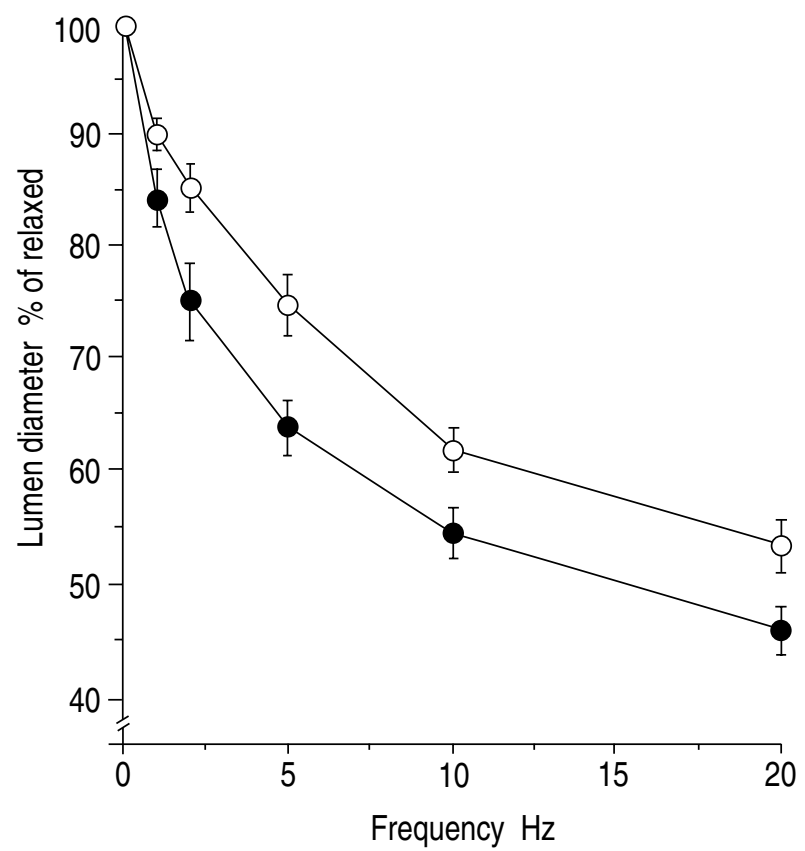

Fig. 5. - Narrowing of airways to electrical field stimulation (0.1 $\mathrm{ms}, 15 \mathrm{~V})$. The large airway (- - narrowed significantly more than the small airway $\left(-\mathrm{O}_{-}\right)(\mathrm{p}<0.05)$. Narrowing is expressed as the percentage of relaxed lumen diameter. 
measured. Lung liquid movement was observed in the majority of airways viewed, and, in general, was seen moving in one direction during narrowing and reversing during relaxation in response to an agonist or EFS. The direction was presumably indicative of the pressure gradient in the fluid which developed during narrowing. If a small proximal branch was sectioned to provide relief of the pressure in the airway under observation, then the fluid was seen to move toward the opening with some exiting during narrowing and returning during relaxation.

EFS, using platinum electrodes placed across the base of the trachea, produced rapid constriction $(<7 \mathrm{~s}$ to maximum response) of most airways excepting some distal bronchioles (L4-5, $2^{\circ}$ ) at short pulse durations (0.1-0.2 $\mathrm{ms}, \sim 30 \mathrm{~V})$. By shifting the electrodes to bridge a main stem bronchus, more of the distal airways were activated $(0.1 \mathrm{~ms},<20 \mathrm{~V})$. Figure 5 shows that the extent of narrowing was approaching a maximum at $20 \mathrm{~Hz}$, where the lumen of large airways constricted to $46 \pm 2.1 \%(n=5)$, significantly more than those in the small airway group which constricted to $53 \pm 2.2 \%(n=6)$ of initial i.d. $(p<0.05)$. The airways relaxed quickly in less than $15 \mathrm{~s}$. They were blocked by atropine $0.1 \mu \mathrm{M}$, indicating that cholinergic nerves were involved, and by tetrodotoxin $0.3 \mu \mathrm{M}$. Since hexamethonium $1 \mathrm{mM}$ had no effect, it seemed that the EFS was acting on cholinergic nerve endings in the bronchial wall.

The presence of nerves running distally from the carina could be readily demonstrated immunocytochemically. Figure 6 shows several nerve trunks 11-25 $\mu \mathrm{m}$ diameter surrounding bronchioles of 75,115 and $155 \mu \mathrm{m}$ average external diameter (i.e. measured from the outer edge of the smooth muscle layer), and smaller nerve fibre bundles approaching the smooth muscle layer. The smooth muscle was demonstrated concurrently using anti-

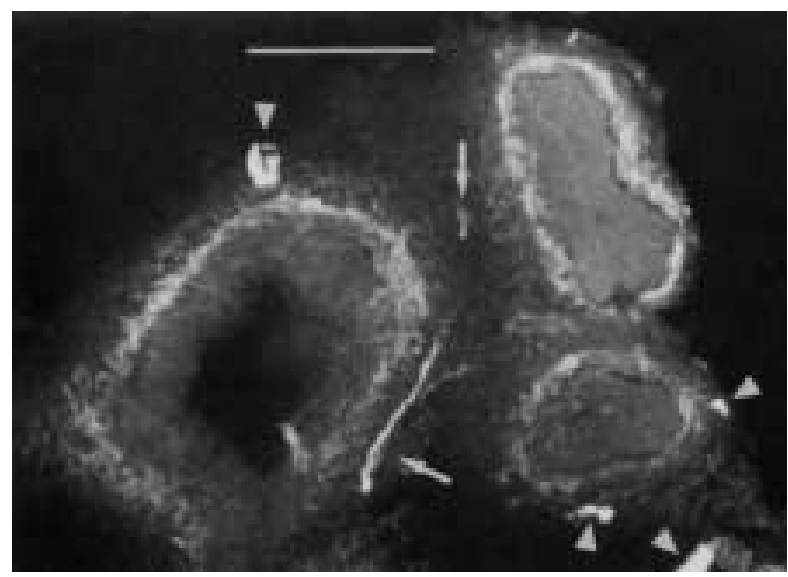

Fig. 6. - Cryostat sections of three branches of an airway from a foetal bronchial tree, that have been stained concurrently for nerve trunks (arrowheads) and fibre bundles (arrows) with the $68 \mathrm{kDa}$ neurofilament antibody (FITC conjugate), and for smooth muscle using smooth muscle myosin (rhodamine red conjugate). The FITC nerve staining is shown, but some of the intense rhodamine red signal has passed through the FITC filter showing the smooth muscle. Overexposure during photography has enabled the mucosal layer and the mesenchymal matrix to be seen in the background but causing the nerve trunks to be slightly blurred. (Scale bar 100=um). FITC: fluorescein isothiocyanate. a)

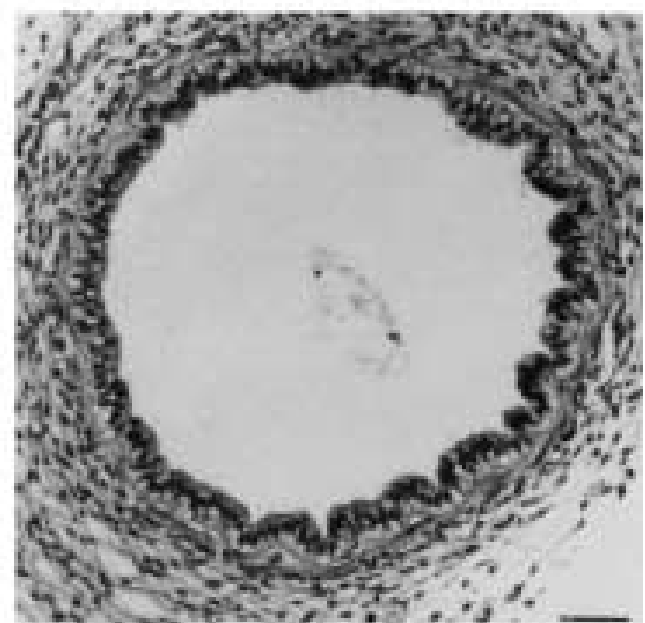

b)

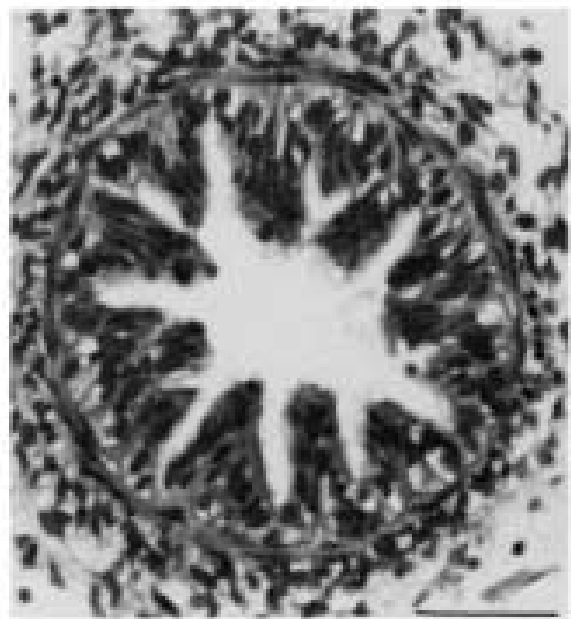

c)

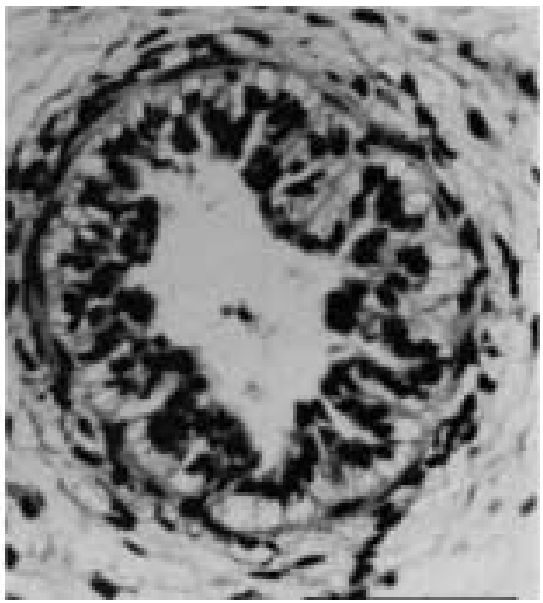

Fig. 7. - Haematoxylin and eosin (H\&E) stained sections of airways from a foetal bronchial tree. a) A small airway almost relaxed, showing the extracellular matrix becoming more condensed surrounding the bronchiole. b) A small airway maximally narrowed with acetylcholine (ACh) showing extensive mucosal folding. c) A smaller airway partially contracted as the folds in the mucosa show. The smooth muscle layer is $1-2$ cells thick. (Scale bar=50 $\mu \mathrm{m}$ ). 
bodies either to smooth muscle myosin (fig. 6) or $\alpha$-actin (not shown), and by over-exposure during photography the mucosa and the mesenchymal supporting matrix is also shown in the background.

In fixed, paraffin embedded sections stained with $\mathrm{H} \& \mathrm{E}$ the loose extracellular matrix is seen becoming more condensed around the airway wall. The density increases with increasing airway size (fig. $7 \mathrm{a}-\mathrm{c}$ ). The mucosa comprises mainly epithelial cells, with a little submucosa in the larger airways. Figure 7 a shows a relaxed small airway, while figure $7 \mathrm{~b}$ shows a small airway maximally narrowed by $\mathrm{ACh}$ with extensive folding of the mucosa. Figure $7 \mathrm{c}$ shows a smaller airway, partially contracted, with a smooth muscle layer 1-2 cells thick.

\section{Discussion}

This study shows that narrowing responses are well developed in the airways of the lungs of 20-35 g pig foetuses (40-45 days gestation); from the main stem bronchi to the terminal regions $(20-35 \mu \mathrm{m}$ i.d.) of the late generation 3rd order branches. Bronchoconstriction to micromolar concentrations of $\mathrm{ACh}$, histamine and substance $\mathrm{P}$ indicated that their receptors were present on the smooth muscle, whilst inhibition of narrowing with $\beta$-adrenoceptor agonists was evidence of $\beta$-receptors. Strong responses to $\mathrm{K}^{+}$depolarizing solution showed that the smooth muscle membrane was polarized. The remarkable display of spontaneous narrowing in the airways of many of the bronchial tree preparations indicated that the membrane potential could fluctuate readily, possibly as a result of stretching of the smooth muscle by an increase in luminal pressure. Blockade of spontaneous contractions and of $\mathrm{K}^{+}$depolarizing responses by verapamil, a blocker of voltage-operated $\mathrm{Ca}$ channels, indicated that current carried by $\mathrm{Ca}^{2+}$ was likely to be involved in these responses. In short, the smooth muscle of the bronchial tree showed similar characteristics to that seen in the postnatal pig [15, 16]. Moreover, strong narrowing responses to EFS were seen, which were blocked by atropine, indicating that a functional cholinergic nervous supply was present.

The small bronchioles showed both a greater narrowing (reactivity) and sensitivity to ACh than did the large airways. The latter had thicker walls that were possibly less compliant, thereby limiting the extent of narrowing. In the small airways, a greater wall compliance would require less tension in the wall to initiate shortening, so that a lower threshold concentration of ACh would be needed. A contribution to this increased sensitivity from the effect described by La Place's Law $(\mathrm{P}=2 \mathrm{~T} / \mathrm{r})$, i.e. the inward directed narrowing force $(\mathrm{P})$, arising from the tension $(\mathrm{T})$ in the wall produced by the contracting smooth muscle, is inversely related to the radius (r), is difficult to assess. This is because in the larger airways the thickness of the smooth muscle layer increases, thereby increasing $\mathrm{T}$, but the relationship between $\mathrm{T}$ and $\mathrm{r}$ has not been determined. It is also possible that the smooth muscle is intrinsically more sensitive in the smaller airways, although in adult airways the sensitivity of the smooth muscle does not change in the bronchial tree [16]. All airways in the present study showed an extensive occlusion of the lumen from folding of the mucosa, often produced by only a few layers of smooth muscle cells in their wall. The lesser occlusion in the large airways was probably a reflection of the larger diameter [17] and a stiffer wall. A greater sensitivity and reactivity of smallbore (1-2 mm i.d.) vs large-bore (4-6 $\mathrm{mm}$ i.d.) airways has also been shown in adult airways [18], where mucosal folding and wall properties have also been implicated in experiments in which narrowing was measured indirectly by changes in flow.

Airways narrowed quickly in response to EFS, but the extent of narrowing was less than that seen with ACh or $\mathrm{K}^{+}$depolarizing solution, and the large ones narrowed slightly more than small ones. This may have been due to a lesser development of the innervation to the smaller bronchioles. Nerve trunks with fibre bundles skirting the smooth muscle could be readily detected lying close to small and large airways, and nerve bundles were regularly observed in the vicinity of smaller airways, $>60 \mu \mathrm{m}$ i.d., but we could not be sure that they were necessarily innervating the adjacent smooth muscle, nor that neurotransmitter was stored in the varicosities and that a full complement could be released. The EFS responses were atropine and tetrodotoxin sensitive, but were unaffected by hexamethonium, the latter indicating that the responses were not occurring via ganglia-mediated transmission in the nerve trunks. Stimulation took place in the close vicinity of the large nerve trunks present at the base of the trachea, but we were unable to elicit narrowing responses via the nerve trunks (not shown), so it seemed likely that they had been disrupted during the dissection procedure to remove the vasculature and parenchyma.

Dynamic airway narrowing could be followed in these foetal airways because they were translucent, with the light passing through the body of the airway. The walls could be readily distinguished, possibly because the wall curvature increased the distance that the light had to pass through from below. The large and abundant nuclei in the epithelial layer may also have added to the light absorbed on the inner wall edge. In large airways, where the wall was thicker, the light transmitted through the body of the airway was diminished and the whole airway appeared darker. Wall edge detail decreased and the outer edge of the wall was not clearly defined. Here, the extracellular matrix was more heavily condensed onto the smooth muscle (see fig. 7a), further reducing the definition of the outer wall edge. However, the potential to follow the folding of the mucosa exists in these medium to large airways. Here, the mucosal folds are wider and folding could be seen as parallel ridges becoming more distinct as the airway narrowed (not shown). It is just possible to see the beginnings of mucosal folding in figure $2 \mathrm{e}$. With further attention to the intensity and direction of the beam(s) of transmitted light and greater removal of the extracellular matrix surrounding the airways, further improvements to visualization of the airway wall and lumen 
should be possible. The validity of this novel technique of measuring the lumen diameter and wall thickness was checked by showing, firstly, that there was no change in the area of the wall as its thickness increased during narrowing; and, secondly, that these parameters in relaxed airways agreed closely with those obtained by morphometric measurements of sections of the same airways subsequently fixed, cryo-sectioned and H\&E stained (see Methods).

What is the purpose of these narrowing responses of foetal airways - either to stimuli or spontaneously? It is tempting to speculate that spontaneous activity serves to move the lung fluid along the airways in order to maintain an even positive pressure, particularly in localized areas of the bronchial tree. Well regulated pressure in the airways would enable the growth of the lung tissue to proceed evenly. Spontaneous breathing movements of the foetus have an overall effect in shifting lung fluid towards the mouth, but they are unlikely to exert a localized effect on the pressure of the lung fluid in a particular airway. This positive intraluminal pressure maintained by the foetal glottis is necessary for normal lung growth [1]. Furthermore, the cellular debris, which is consistently seen in all sizes of airways, is observed to move to and fro in the lumen. Presumably, this movement prevents the debris from settling, thereby reducing any tendency for it to obstruct the lumen of small airways. A preliminary inspection of the lung liquid indicates that it predominantly contains nuclei which have lost their cytoplasm. They appear to be representative of monocytes, macrophages, lymphocytes and epithelial cells. These presumably come through the airway wall, but the possibility of cellular material arising from the lumen of the differentiating epithelial bud cannot be excluded.

The spontaneous bronchomotor tone reported here in the in vitro bronchial tree of the prenatal lung now needs to be demonstrated in the intact lung in vivo. Toward this objective, it would be predicted that the pressure waves transmitted by the lung liquid arising from the spontaneous narrowing of the airways might be detectable at the level of the trachea in the lung in situ. Also, the rate of lung liquid secretion needs to be measured in the first trimester foetus to show that it is significant, albeit adequate to maintain the volume of the lumen of the airways at slightly positive pressure.

Acknowledgements: The authors thank the staff of the Watsonia Abattoir for their co-operation and support in providing the foetal pigs.

\section{References}

1. Harding R, Dickson KA, Hooper SB. Fetal breathing, tracheal fluid movement and lung growth. In: Gluckman, Johnson, Nathaniel, eds. Advances in Fetal Physiology. Ithaca, Perinatology Press, 1989; pp. 153-175.

2. Booth RJ, Sparrow MP, Mitchell HW. Early maturation of force production in pig tracheal smooth muscle during fetal development. Am J Respir Cell Mol Biol 1992; 7: $590-597$.

3. Lewis M. Spontaneous rhythmical contraction of the muscles of the bronchial tubes and air sacs of the chick embryo. Am J Physiol 1924; 68: 385-388.

4. Schopper W. Embryonales und erwachsenes Lungengewebe vom Meerschweinchen und Huhn in der Kulter mit Zeitrafferbeobachtungen an Flimmerepithel, sog. Alveolarphagocyten und von Kontraktionen der Bronchialmuskulatur. Virchows Arch 1935; 295: 623-644.

5. Schopper W. Uber das Verhalten des Lungengewebes in der Gewebekultur (Filmdemonstration). Arch Exp Zellforsch 1937; 19: 326-328.

6. McCray PB. Spontaneous contractility of human fetal airway smooth muscle. Am J Respir Cell Mol Biol 1993; 8: $573-580$.

7. Flint JM. The development of the lungs. Am J Anat 1906; 6: 1-136.

8. Weibel E. Design and development of the mammalian lung. In: Weibel ER, eds. The Pathway for Oxygen. Cambridge MA, Harvard University Press, 1984; pp. 211-230.

9. Hirai Y, Takebe K, Takashina M, Kobayashi S, Takeichi M. Epimorphin: a mesenchymal protein essential for epithelial morphogenesis. Cell 1992; 69: 471-481.

10. Mitchell JJ, Reynolds SE, Leslie KO, Low RB, Woodcock-Mitchell J. Smooth muscle cell markers in developing rat lung. Am J Respir Cell Mol Biol 1990; 3: 515-523.

11. Woodcock-Mitchell J, White S, Stirewalt W, Periasamy M, Mitchell J, Low RB. Myosin isoform expression in developing and remodelling rat lung. Am J Respir Cell Mol Biol 1993; 8(6): 617-625.

12. Pomeroy RN. Infertility and neonatal mortality in the sow. III. Neonatal mortality and foetal development. $J$ Agric Sci 1960; 54: 31-56.

13. Altman PL, Dittmer DS, eds. Biology Data Book. Federation of American Societies for Experimental Biology, Bethesda, MD. 2nd Edn, 1972; pp. 176-182.

14. Mohammad MA, Sparrow MP. The distribution of heavychain isoforms of myosin in airways smooth muscle from adult and neonate humans. Biochem J 1989; 260: 421426.

15. Mitchell HW. Electromechanical effects of tetraethylammonium and $\mathrm{K}^{+}$on pig isolated tracheal smooth muscle. Lung 1987; 165: 129-142.

16. Sparrow MP, Mitchell HW. Contraction of smooth muscle of pig airway tissues from before birth to maturity. J Appl Physiol 1990; 68: 468-477.

17. James AL, Paré PD, Hogg JC. The mechanics of airway narrowing in asthma. Am Rev Respir Dis 1989: 139: 242-246.

18. Mitchell HW, Sparrow MP. Increased responsiveness to cholinergic stimulation of small diameter cartilaginous bronchi compared to large. Eur Respir J 1994; 7: 298-305. 